resolution. Further studies to investigate these changes may help reveal potential therapeutic targets for $\mathrm{AH}$.

\section{P027 RETROSPECTIVE ANALYSIS OF BONE PROTECTION CARE IN PRIMARY BILIARY CHOLANGITIS (PBC) IN A DISTRICT GENERAL HOSPITAL}

Yun Jie Chew*, John Hutchinson. Mid York NHS Trust, UK

\subsection{6/gutjnl-2021-BASL.36}

Introduction We performed a review of bone management and follow up among patients with Primary Biliary Cholangitis (PBC) at Mid Yorkshire Hospitals NHS Trust (MYHT). MYHT provides hospital services to a population of 530,000. Methods We undertook a retrospective analysis of 542 patient records and clinic letters through Cito, which is the Trust's electronic Document Management System and ICE system. We included patients who were under the outpatient care of both the Hepatology and Gastroenterology services over a 3 year period from December 2017 to December 2020.

Results In term of bone care, 54\% $(n=21)$ had up to date bone density scan (DEXA), 33\% $(n=13)$ have never had a DEXA scan and 13\% $(n=5)$ have had DEXA scan in the past but were due for a repeat scan. Among those who had a DEXA scan ( $n=26$ and 67\%), 42\% $(n=11)$ of them had osteoporosis and 31\% $(n=8)$ had osteopenia.

In respect to $\mathrm{PBC}$ patients diagnosed with osteoporosis or osteopenia, only $10 \%(n=2)$ were not on any bone protection therapy. This includes bisphosphonates, calcium and vitamin D replacement. However, depending on the serum level of calcium and vitamin D of patient, they are able to get calcium and vitamin D over the counter and therefore not prescribed by hospital or GP. Calcium and Vitamin D replacement is essential since there is sufficient evidence suggesting calcium and vitamin D alone prevent bone loss and reduce fracture. ${ }^{1}$ Discussions It was recommended by National Institute for Health and Care Excellence (NICE) that after a minimum of 2 years, risk assessment need to be undertaken among the population whose original calculated FRAX score was in the region of the intervention threshold for treatment. It was unsure whether they have had a risk assessment for osteoporosis since the Fracture Risk Assessment Tool (FRAX) score was not documented in clinic letters. ${ }^{2}$ Therefore, we need to ensure risk assessment for osteoporosis is performed in the clinic and documented clearly in the clinic letter using the FRAX score.

\section{REFERENCES}

1. Sunyecz JA. The use of calcium and vitamin $D$ in the management of osteoporosis. Ther Clin Risk Manag 2008;4(4):827-836. doi:10.2147/tcrm.s3552

2. National Institute of Health and Care Excellence (NICE). Osteoporosis: assessing the risk of fragility fracture [CG146] 2017. Available from: https://www.nice.org. uk/guidance/cg146 [Accessed 28th May 2021]

\begin{tabular}{ll} 
Abstract P027 Table 1 & \\
\hline & Number of patients $(\mathrm{n}=39)$ \\
\hline Bone protection & 12 \\
Osteoporosis & 8 \\
Osteopenia & 7 \\
Normal & \\
\hline
\end{tabular}

\section{P028 CHRONIC LIVER DISEASE, PLATELET TRANSFUSION \& QUALITY OF LIFE - A BRITISH LIVER TRUST PATIENT- SURVEY}

'Jonathan Worsfold, 'Vanessa Hebditch, ${ }^{2}$ Stephen Ryder. 'British Liver Trust, UK; ${ }^{2}$ Queen's Medical Centre, Nottingham, UK

\subsection{6/gutjnl-2021-BASL.37}

Introduction For patients with chronic liver disease, thrombocytopenia is a common complication that impacts on management of cirrhosis and has implications for planned surgical/ diagnostic procedures due to an increased risk of bleeding. Platelet transfusion (PT) has been the standard of care for management of thrombocytopenia. There is a need to better understand the views and experiences of UK patients with advanced disease and how their condition, and requirements for PT, affect quality of life.

Method The British Liver Trust (BLT) undertook a patient survey between December 2020 and February 2021. Patients were invited to participate via BLT channels (monthly newsletter; patient support groups) answering 30 questions (anonymously) via the Survey Monkey platform. The survey was conducted independently by the BLT, supported by an educational grant from Shionogi.

Results A total of 121 patients completed the survey. Alcohol related liver disease was the most commonly reported primary liver disease $(28 \%) ; 63 \%$ of respondents were female; $73 \%$ were aged over 45 years, and $51 \%$ have been told they may require a liver transplant in the future. A large proportion $(69 \%)$ of patients reported that liver disease sometimes affects their ability to think clearly. Half of responders recorded having to take time off work for liver-related hospital appointments; $40 \%$ travel $>25$ miles to their liver centre/hospital for routine appointments, and 55\% said that the COVID-19 pandemic had led to procedures related to their liver disease being cancelled. Many patients (62\%) reported that healthcare professionals (HCPs) have difficulties inserting a needle into a vein and $40 \%$ noted that they have needed a dental extraction since being diagnosed. Almost a third (30\%) have required a PT at some time, and of those, $70 \%$ had to stay in hospital the night before the PT. Patients reported that the reasons for needing a PT were typically well-explained. Among recipients of $\mathrm{PT}, 42 \%$ said they felt unwell during or post the PT. Following completion of the survey, 33 patients provided consent for voluntary follow-up and supplementary questions from the BLT.

Conclusions A survey of patients with chronic liver disease highlights that the COVID-19 pandemic has impacted on and delayed planned procedures, and identifies that both chronic liver disease, and the requirement for PT, pose a burden to patients that may adversely affect quality of life.

\section{P029 PLASMALEMMA VESICLE-ASSOCIATED PROTEIN (PLVAP) MEDIATES MONOCYTE TRANSMIGRATION ACROSS HUMAN HEPATIC SINUSOIDAL ENDOTHELIUM IN RESPONSE TO THE SENESCENT SECRETOME}

${ }^{1}$ Alex Wilkinson*, ${ }^{1}$ Daniel Patten, ${ }^{1}$ Sam Hulme, ${ }^{2}$ Matthew Hoare, ${ }^{1}$ Shishir Shetty. ${ }^{1}$ University of Birmingham, Institute of Immunology and Immunotherapy, UK; ${ }^{2}$ University of Cambridge, Department of Medicine, UK

10.1136/gutjnl-2021-BASL.38 
Senescence is an autonomous state of cell cycle arrest which is an important mechanism preventing malignant transformation. Recent evidence suggests that cellular senescence may also have a double-edged effect, contributing to chronic liver diseases (CLDs) and hepatocellular carcinoma (HCC). Senescent cells release a secretome, known as the senescence-associated secretory phenotype (SASP), which regulates the clearance of senescent cells through the recruitment of leukocytes from the circulation. We hypothesised that the SASP modulates leukocyte recruitment by regulating the phenotype of human hepatic sinusoidal endothelial cells (HSEC), the gatekeeper for leukocyte homing to the liver. To elucidate the underlying mechanisms, we undertook qPCR of SASPtreated HSEC and found that plasmalemma vesicle-associated protein (PLVAP) was transcriptionally regulated by SASP treatment. PLVAP is an endothelial-specific molecule recently highlighted in single-cell sequencing studies and has previously been implicated in monocyte trafficking during foetal development.

PLVAP expression was studied in human patient samples at the gene and protein level via qPCR and immunohistochemistry. To understand the effects of the SASP on PLVAP expression, HSEC were isolated from human livers by immunomagnetic selection and subject to 24-hour treatment with conditioned medium from RAS-senescent IMR90 fibroblasts (RAS-CM). PLVAP expression was then determined by qPCR and immunocytochemistry followed by high-content imaging. We observed strong PLVAP expression on endothelium in CLD and HCC patients, and confirmed that PLVAP expression in vitro is upregulated in primary HSEC by the senescent cell secretome.

To study the effects of RAS-CM treatment on monocyte recruitment and the contribution of PLVAP, we undertook flow adhesion assays under physiologically low shear stress with primary HSEC and monocytes isolated from peripheral blood. RAS-CM promoted monocyte adhesion and transmigration across HSEC. To investigate whether PLVAP plays a role in SASP-mediated monocyte recruitment, flow assays were performed with siRNA-treated or anti-PLVAP antibodyinhibited HSEC. We found that the proportion of adhered monocytes which underwent transendothelial migration was significantly impaired in PLVAP-inhibited HSEC. Following flow assays, cells were then fixed and analysed by confocal microscopy. We demonstrated that monocyte transmigration occurred predominantly via the paracellular route, in association with CD31 and independently of intercellular adhesion molecule 1, with PLVAP closely associating with the actin cytoskeleton. These results demonstrate that PLVAP plays an important role in SASP-driven monocyte recruitment across human HSEC. Furthermore, PLVAP expression in CLD and HCC may be driven by tissue senescence and could be a novel approach to target aberrant monocyte recruitment in liver disease.

\section{P030 OUTCOMES OF INCOMING AND OUTGOING SECOND OPINIONS FROM A UK LIVER TRANSPLANT CENTRE - A ROADMAP FOR PAN-UK CENTRE DATA COLLECTION}

\footnotetext{
${ }^{1,2}$ Alexander Boyd ${ }^{*},{ }^{1}$ Sara Mahgoub, ${ }^{1}$ Sean Morris, ${ }^{1}$ Alessandro Parente,

1,2 Matthew Armstrong, ${ }^{1,2}$ Neil Rajoriya. 'University Hospitals Birmingham NHS Foundation Trust, Birmingham, UK; '2 University of Birmingham, Birmingham, UK
}

Background Second (2nd) transplant centre opinions for patients declined for liver transplantation (LT) are considered a rarely needed, but important component of the assessment process. There remains a lack of published data pertaining to the numbers of patients referred for 2 nd opinions and their outcomes in the UK.

Aim To identify and track outcomes of patients referred into our LT centre (Birmingham, UK) for 2nd opinions (INCOMING) and those referred to a different centre (OUTGOING) between January 2012 - December 2020.

Methods INCOMING: all new outpatient referrals to the Birmingham LT Unit from out-of-region centres $(n=1751)$ were reviewed and requests for 2 nd opinions were collated. OUTGOING: patient records of those not listed at the Birmingham LT Unit $(n=426)$ were reviewed to identify those referred for 2 nd opinion to one of the 6 other UK LT centres. NHS identification numbers were cross-checked with NHSBT regarding LT elsewhere.

Results INCOMING: 23 2nd opinions (17 Leeds, 3 Royal Free, 1 Edinburgh, 1 Cambridge, 1 King's) were provided by Birmingham. Median age was 53 and the commonest aetiology was alcohol related liver disease (ArLD) $(n=15 ; 65 \%)$. The risk of alcohol addiction/relapse was the main reason for initial LT refusal in 8 of these cases. Of the 2 nd opinions, 13/ $23(57 \%)$ patients remained unsuitable for LT after review and case discussion The remaining 10/23 (43\%) underwent a formal LT assessment, of whom 5 were deemed 'too high risk', 3 listed (1/3 subsequently transplanted) and 2 died preassessment.

OUTGOING: 8 Birmingham patients (aetiologies NAFLD, ArLD, LT graft loss, Budd-Chiari, PSC, venous thrombosis) were referred to other LT centres (4 King's, 2 Royal Free, 1 Cambridge, 1 Leeds) for a 2 nd opinion. Of these, $3 / 8$ (38\%) were assessed and listed for LT by King's (1 transplanted, 2 waiting), 2 were assessed and declined LT, 2 were unsuitable for assessment and 1 died before being seen.

Cumulatively, only 6/31 (19\%) of 2nd opinions were deemed suitable to list for LT.

Conclusions Requests for a 2nd centre LT opinion are rare. The majority $(>80 \%)$ are still deemed 'too high risk' (alcohol, medical/anaesthetic risk) for LT after a 2 nd LT centre opinion. There is a large discrepancy between the numbers of patients declined LT in the UK and the number of 2 nd opinions sought. Further work is required to understand the reasons for this. A national UK LT assessment database would enable greater LT decision making.

\section{P031 THE IMPLEMENTATION OF THE NATIONAL HEALTH SERVICE ENGLAND SERVICE FOR HEPATITIS C ANTIBODY TESTING IN COMMUNITY PHARMACIES: A NATIONAL SURVEY OF HEPATITIS C OPERATIONAL DELIVERY NETWORKS}

${ }^{1}$ Charlotte Cook*, ${ }^{2}$ Leila Reid, ${ }^{2}$ Stuart Smith, ${ }^{3}$ Deborah Crockford, ${ }^{1}$ Salim Khakoo, ${ }^{1}$ Julie Parkes, ${ }^{1}$ Ryan Buchanan. ${ }^{1}$ University Of Southampton, UK; ${ }^{2}$ Hepatitis C Trust, UK; ${ }^{3}$ Community Pharmacy South Central (Hants. and Isle of Wight LPC), UK

\subsection{6/gutjnl-2021-BASL.40}

Introduction To meet and maintain World Health Organisation Hepatitis C (HCV) elimination target, it is essential that testing is scaled up and targeted at high-risk individuals. In September 2020 NHS England (NHSE) commissioned an advanced service to test people who inject drugs for HCV in 